\begin{tabular}{l|l} 
Reflexiones & $\begin{array}{l}\text { Revista Colombiana } \\
\text { de Educación, N. } 62 \\
\text { Primer semestre de 2012, } \\
\text { Bogotá, Colombia. }\end{array}$
\end{tabular}

\section{Escuela, memoria y conflicto en Colombia. Un ejercicio del estado del arte de la temática}

\author{
//School, memoirs and conflict in \\ Colombia. A brief approach of the \\ state of the art
}

//Escola, memoria e conflito na Colômbia. Um exercício de estado da arte sobre a temática

\author{
Absalón Jiménez Becerra* \\ Raúl Infante Acevedo** \\ Ruth Amanda Cortés***
}

Recibido: 07/09/2011 Evaluado: $02 / 12 / 2011-28 / 12 / 201$

Doctor en Educación del Doctorado Interinstitucional en Educación, Universidad Distrital Francisco José de Caldas, magister en Historia de la Universidad Nacional de Colombia, magister en Estudios Políticos de la Pontificia Universidad Javeriana y licenciado en Ciencias Sociales de la Universidad Pedagógica Nacional. Docente-investigador de la Maestría en Investigación Social Interdisciplinaria-MISI de la Universidad Distrital de Bogotá. Correo electrónico: abjibe@hotmail.com

Licenciado en Ciencias Sociales de la Universidad Pedagógica Nacional. Docente-investigador de la Licenciatura en Educación Comunitaria y Derechos Humanos de la Universidad Pedagógica Nacional y docente de la Fundación universitaria Los Libertadores. Correo electrónico: raulinfanteacevedo@yahoo.com

Candidata a Doctora en Educación de la Universidad Pedagógica Nacional de Colombia, magister en Investigación Social UPN-CINDE, especialista en Enseñanza de la Historia de la Pontificia Universidad Javeriana y licenciada en Ciencias Sociales de la Universidad Pedagógica Nacional. Profesional de la subdirección académica del Instituto para la Investigación Educativa y el Desarrollo Pedagógico-IDEP. Correo electrónico: amandacortes2006@yahoo.es

\section{Palabras Clave}

Memoria, conflicto, escuela, experiencia, narrativa, subjetivación. 


\section{Abstract}

The main topic of this paper is memoir as a teaching tool which in turn becomes a teaching experience related to stress and systematization. A fight for an official memoir is evident in school, specifically working subjects, such as History and Social Sciences, where official standards follow policies of the institutional memoir as well as policies of the state history. However, currently some school teachers have stressed memoir angles and the official story through newer and meaningful experiences of educational tasks where the topic of social, political and armed conflict both in the rural and urban scenarios has become a possibility of work in the classroom.

\section{Keywords}

Memoir, conflict, school, experience, narrative, subjectivation.

\section{Resumo}

O fio condutor do presente artigo é a memória, que como dispositivo de trabalho pedagógico se converte ao mesmo tempo em um elemento de tensão e sistematização da experiência docente. A luta pela memória oficial se evidencia na escola, particularmente nas disciplinas como História e Ciências Sociais escolares, que, ao seguirem uma linha oficial, inscrevem-se nas políticas da memória institucional, nas políticas da história oficial do Estado. Não obstante, nos últimos anos, alguns professores tem conseguido tensionar os ângulos da memória e da história oficial, recorrendo a experiências significativas e de inovação de trabalho pedagógico, onde o tema do conflito social, político e armado, vivido tanto no cenário rural como no urbano, vem se convertendo em uma possibilidade temática de trabalho na sala de aula.

\section{Palavras chave}

Memória, conflito, escola, experiência, narrativa, subjetivação.

\section{Presentación}

En el marco de la Línea de investigación de historia y memoria de la pedagogía del IDEP y del proyecto de investigación Sistematización de experiencias significativas de los docentes de Bogotá en torno al tema de memoria, escuela y conflicto (2011), hemos venido desarrollando un balance en torno a una serie de experiencias puntuales que han abordado el tema de la memoria en contextos escolares. Para tal efecto, hemos tenido como trasfondo dos situaciones particulares: en primer lugar, las iniciativas que en el ámbito universitario e institucional, particularmente sus facultades de educación en coordinación con el Instituto para la Investigación Educativa y el Desarrollo Pedagógico- IDEP, desde 
finales de los años noventa y primera década del siglo XXI, han abordado el tema de la memoria, primero de manera tangencial $y$, luego, de manera explícita; y en segundo lugar, la constitución de la Red Interinstitucional de Memoria por parte del Instituto para la Paz, la Pedagogía y el Conflicto Urbano- Ipazud, de la Universidad Distrital Francisco José de Caldas de Bogotá, en el año $2007^{1}$. Alrededor de estas dos experiencias se han aglutinado una serie de iniciativas de trabajo que a nivel local se constituyen en un insumo fundamental para dar cuenta de la experiencia docente con relación el tema de la memoria en la escuela.

En términos metodológicos, la elaboración del estado del arte que a continuación se presenta es un ejercicio pertinente para dar cuenta de la memoria como un fenómeno emergente en la escuela. También tenemos que anotar que el presente ejercicio debe ser tenido en cuenta

Particularmente, en la Universidad Distrital Francisco José de Caldas de Bogotá, debemos reconocer la iniciativa del Instituto para la Paz, la Pedagogía y el Conflicto Urbano, Ipazud, en torno a la constitución de la Red Interinstitucional sobre el tema de Memoria, desde el año 2007. En esta iniciativa se han logrado publicar algunos trabajos, de manera dispersa, sobre el tema de escuela y memoria. Algunos de los artículos que nutren el presente estado del arte se encuentran en una serie de libros gestionados por el Instituto: Memoria y conflicto. Cátedra de ciudadanía (2008), compilado por Adrián Serna; Las luchas por la memoria (2009), compilado por Absalón Jiménez B.; El papel de la memoria en los laberintos de la justicia, verdad y reparación, (2009) compilado por Adrián Serna y Diana Gómez; y Los crisoles de la memoria. Propuestas teóricas, metodológicas y estratégicas para el estudio de la memoria (2009), compilado por Adrián Serna. bajo los parámetros propios de lo que es un ejercicio de carácter investigativo. De tal manera, como de tiempo atrás lo hemos dado a conocer, el estado del arte se puede pensar desde tres perspectivas: en primer lugar, como un ejercicio de apropiación del conocimiento, en este caso en torno al tema de la memoria en la escuela; en segundo lugar, como un ejercicio "de investigación de la investigación"; y en tercer lugar, como un ejercicio fundamental para dar un paso a lo inédito y novedoso en torno al abordaje de un tema en particular, facilitando a la vez la delimitación de una pregunta investigativa (Jiménez, B., 2004 ).

Nuestro interés, en el presente estado del arte, visto como un ejercicio de "investigación de la investigación", es contextualizar las experiencias en torno al tema de la memoria en la escuela, preguntándonos desde qué momento aparece el concepto de memoria en la escuela y bajo qué condiciones los docentes en ejercicio desarrollan esta serie de iniciativas de trabajo; en segundo lugar, buscamos dar cuenta de las perspectivas conceptuales y metodológicas por medio de las cuales los docentes en ejercicio han abordado el tema; y por último, evidenciar los diversos niveles de avance de las experiencias.

Por lo demás, nuestro interés es valorar los ejercicios que han asumido el tema de la memoria en la escuela como parte de un 
"dispositivo" 2 de intervención pedagógico. En este sentido, valoramos cierto tipo de experiencias que ponen a disposición el tema de la memoria para desarrollar un conjunto de actividades escolares con un interés en particular y en una relación de poder: cuestionar el relato oficial y/o construir y valorar esos otros relatos y esas otras memorias no valoradas por la escuela. Mediante el dispositivo de intervención pedagógico se aborda un objeto contemporáneo de preocupación en la escuela, la memoria, la cual se puede rescatar desde la misma cotidianidad de los sujetos escolares, ser abordada desde diversas metodologías, trabajada desde diversas fuentes, ambientada con diversas ayudas didácticas $y$, en general, acompañada de experiencias propias del sujeto docente. Experiencias que se constituyen de manera implícita en una determinada concepción de práctica y saber pedagógico.

La memoria como dispositivo de trabajo escolar y como sistema simbólico no reconocido de manera formal en el currículo, propicia el desarrollo de una serie de habilidades cognitivas e intersubjetivas que nos permiten comprender de una manera significativa, entre otros temas, el conflicto social y político por el cual han atravesado los colombianos. De tal manera, algunos docentes en ejercicio, con relación al tema de la memoria, visto como dispositivo de intervención pedagógico, han organizado de manera implícita un contenido temático, establecido una secuencia de actividades, unas técnicas de trabajo, han planteado un tema y constituido un pregunta guía, han logrado el agrupamiento de un colectivo de estudiantes y han organizado una serie de actividades en torno a la memoria en el contexto escolar, ya sea con base en un hecho o acontecimiento que ha marcado la historia nacional, un tema de carácter histórico formal, una experiencia desgarradora con relación al conflicto armado o con relación al contexto en el que se encuentra ubicada la escuela, entre otros.

En el ámbito conceptual, en el marco del proyecto de sistematización de experiencias que nos encontramos desarrollando

2 Por dispositivo pedagógico se podrían designar además, en términos generales, los operadores materiales de poder, es decir, técnicas, estrategias, y formas de sujeción instaladas por el poder escolar. Desde esta perspectiva se insiste en la importancia de los mecanismos de dominación, los cuales son de naturaleza heterogénea, se trata tanto de discursos como de prácticas, de instituciones como de acondicionamientos arquitectónicos, teniéndose en cuenta, inclusive, tanto lo dicho como lo no dicho. En general, los análisis en torno al tema del dispositivo se inspiran en Foucault, M. (1976). Historia de la sexualidad. La voluntad de saber, tomo I. México: Siglo XXI. 
valoramos la memoria como parte de las relaciones de significado del sujeto, como una construcción simbólica y subjetiva, y como un ejercicio de evocación del pasado y representación de lo ausente ( $\mathrm{Ri}$ coeur, 2000). En buena medida, el proyecto de investigación "Escuela, memoria y conflicto. Una mirada desde la experiencia y narrativa del docente" (IDEP, 2011), parte de la premisa básica de la representación del pasado y la evocación de lo ausente mediado por la memoria, la experiencia del docente en ejercicio y la manera como ha asumido el tema del conflicto, como un tema posible de abordar en el aula de clase en la ciudad de Bogotá.

En este sentido, el hilo conductor del proyecto es la memoria, que como dispositivo de trabajo pedagógico se convierte a la vez en un elemento de tensión y sistematización de la experiencia docente. La lucha por la memoria oficial se evidencia en la escuela, particularmente en asignaturas como la Historia y las ciencias sociales escolares, que al seguir un lineamiento oficial se inscriben en las políticas de la memoria institucional, en las políticas de la historia oficial del Estado. No obstante, en la actualidad algunos maestros en la escuela, en los últimos años, han logrado tensionar los ángulos de la memoria y la historia oficial recurriendo a experiencias significativas y de innovación de trabajo pedagógico donde el tema del conflicto social, político y armado, vivido tanto en el escenario rural como el urbano, se ha convertido en una posibilidad temática de trabajo en el aula.

De tal manera, mediante el proyecto en mención, nos trazamos como objetivo fundamental la recopilación de experiencias significativas realizadas por parte de los docentes en torno al tema de la memoria y el conflicto desarrollados en el aula, en la ciudad de Bogotá en los últimos años.

Dicho ejercicio académico se hace pertinente en términos sociales y políticos para la ciudad de Bogotá, la Secretaria de Educación y en particular el IDEP, debido a que traza como uno de sus intereses fundamentales el tensionar la memoria en momentos en que el país, desde el año 2005, habla de verdad, justicia y reparación de víctimas, particularmente producto de las últimas experiencias de desmovilización del paramilitarismo en la primera década del siglo XXI. Así mismo, en la ciudad de Bogotá la figura del desplazado se convierte en la más dramática encarnación de nuestros desarraigos. El desplazado con su memoria rota, es la evocación permanente de nuestra propia inestabilidad como proyecto de nación (Sánchez, 2001). Dicha situación a diario la deben afrontar un alto número de docentes en ejercicio en las escuelas públicas de la ciudad en localidades donde reina la exclusión social. 
En el ámbito académico colombiano se hace necesario que la institución escolar sistematice las pocas experiencias significativas que sobre el tema de la memoria logran concretar los maestros en ejercicio. A continuación, bajo los criterios del estado del arte visto como un ejercicio de "investigación de la investigación", damos cuenta de las experiencias más relevantes que sobre el tema de la memoria en la escuela se han destacado en el ámbito local.

El presente estado del arte consta de tres puntos: en el primero, damos cuenta de las experiencias más relevantes que a nivel internacional y nacional han inspirado y dirigido esta iniciativa de trabajo, como la memoria en los contextos escolares; en el segundo, buscamos darle relevancia a las experiencias que han logrado alguna publicación y difusión en forma de libros, capítulos de libro y artículos, los cuales hemos logrado ubicar de tiempo atrás y que se constituyen en un material importante a visibilizar como parte de una temática emergente en la escuela; en el tercero, presentamos las conclusiones del estado del arte en términos de contexto, posturas conceptuales frente al tema, metodológicas y conclusivas.

\section{La memoria en la escuela: experiencias a destacar en Latinoamérica y Colombia}

En el ámbito latinoamericano, las experiencias de las luchas por la memoria, por la historia oficial, las amnistías, los indultos, los perdones y los olvidos, se han extendido a la escuela. Elizabeth Jelin (2002), en la segunda mitad de la década de los años noventa, lidera un proyecto con jóvenes investigadores sobre las memorias de la represión del Cono Sur, en momentos en que los regímenes militares impusieron su "bota", entre los años sesenta y ochenta, en países como Argentina, Chile, Brasil y Paraguay. El programa apunta a la creación de una red de intelectuales públicos de la región, preocupados por el estudio de la memoria societal y demás temas relacionados con ella. El equipo, liderado por Jelin, aborda el tema de la memoria en el plano político, simbólico, personal e histórico. Se trazan tres objetivos: "Primero, entender las memorias como procesos subjetivos, anclados en experiencias y en marcas simbólicas y materiales. Segundo, reconocer a las memorias como objeto de disputa, conflictos y luchas, lo cual apunta a prestar atención al rol activo y productor de sentido de 
los participantes en esas luchas, enmascarados en relaciones de poder. Tercero, historizar las memorias, o sea, reconocer que existen cambios históricos en el sentido del pasado, así como en el lugar asignado a las memorias en diferentes sociedades, climas culturales, espacios de luchas políticas e ideológicas" (Jelin, 2002, p. 2). El grupo se traza el desafío de superar las repeticiones, superar los olvidos y los abusos políticos, con el fin de tomar distancia y al mismo tiempo promover el debate y la reflexión activa sobre ese pasado y su sentido para el presente y el futuro. "Esto implica un pasaje trabajoso para la subjetividad: la toma de distancia del pasado, aprender a recordar. Al mismo tiempo implica repensar la relación entre memoria y política, y entre memoria y justicia" (Jelin, 2002, p. 16).

El esfuerzo del equipo obtiene sus frutos con el texto Educación y memoria (Jelin y Lorenz, 2004). En este se hace un importante esfuerzo por establecer relaciones entre la educación y la memoria, en países como Perú, Chile, Argentina, Uruguay y Brasil. Allí se advierte cómo las versiones de la historia oficial en estos países se han visto confrontadas por otras narrativas históricas, alternativas, aun contradictorias.

Las escuelas fueron un lugar privilegiado por distintos "emperadores de la memoria" que trasladaron a ellas un mandato de memoria que traducía a la vez una exigencia política. En algunos países predominó el silencio. En otros la voz de los sobrevivientes y de los afectados fue una presencia frecuente en actos y actividades escolares

(Jelin y Lorenz, 2004, p. 5). La escuela se constituye en el principal escenario para la materialización de "las políticas de la memoria", las cuales terminan siendo expresión de una memoria e historia oficial por parte de un sector dominante de las sociedades latinoamericanas.

Frente a estas tensiones entre memoria, historia y escuela, en el Cono Sur existe otro ejemplo investigativo a seguir. Para el caso particular de la Argentina, las investigadoras Inés Dussel, Silvia Finocchio y Silvia Gojman (2003), han hecho un esfuerzo importante con escolares de secundaria trabajando la "memoria del horror", con el fin de buscar otras alternativas posibles. Su investigación, Haciendo memoria en el país de nunca más, traza como objetivo que la consigna de Nunca Más se convierta en un acuerdo básico de la sociedad argentina, moviéndose en una lógica de memoria ejemplarizante. La intención de su libro es trabajar el tema de la "memoria del horror" en los contextos escolares, desarrollando desde un inicio una conceptualización básica en torno a los temas de Estado y sociedad y su relación con la democracia y el autoritarismo. En la parte inicial realizan 
un recorrido ágil en torno a la historia de la democracia en Argentina, abordando los diferentes enfoques de la dictadura militar, vivida entre 1976 y 1983. La "memoria del horror" es una memoria situada, desde este periodo hasta inicios del siglo XXI, mediante la cual se desarrolla un balance de la memoria colectiva, las formas y los lugares en los que se sostiene y la relación de los acontecimientos, posteriores a 1983, con los derechos humanos. Por lo demás, la propuesta está acompañada de una serie de sugerencias y un conjunto de actividades para trabajar en el interior de la escuela con los estudiantes. Nunca Más "debe ubicarse en la lista de lo que no debe olvidarse, precisamente porque tiene que ver con la afirmación de un principio básico de cualquier sociedad, que es el de defender el derecho a la vida de todos y cada uno de sus miembros" (Dussel, Finocchio y Gojman, 2003, p. 144).

En el contexto colombiano es para destacar la iniciativa de la profesora Olga Lucía Zuluaga Garcés, quien en el año 2006 intenta activar desde el IDEP la memoria activa del saber pedagógico como categoría de investigación. Desde la perspectiva genealógica y arqueológica en la que se ubican sus reflexiones, el saber pedagógico del maestro, como saber sometido, representó a lo largo de la historia la expresión de una memoria colectiva silenciada. De tal manera, la memoria activa del saber pedagógico debe ser entendida como "el lugar donde podemos encontrar cuáles han sido las formulaciones, las búsquedas, los fracasos, los obstáculos, las continuidades y los avances acerca de la enseñanza... así como las discontinuidades, los discursos, las normas, los reglamentos, los manuales, las disposiciones del cuerpo y los materiales de la memoria" (Zuluaga y Marín, 2006). Para esta investigadora, las posibilidades de comprensión, pero también de emancipación que ofrece una lectura desde el saber pedagógico de aquello en lo que han devenido los sujetos y sus sociedades, se constituye, tal vez, en el valor más importante que adquiere la definición. Esta categoría tiene que ver con el reconocimiento de la memoria individual que los distintos sujetos tienen de las prácticas educativas y formativas de las cuales han hecho parte como maestros, estudiantes, ciudadanos y/o miembros de una institución social. Para la profesora Zuluaga, un saber pedagógico, que se constituye en recuerdo y expresión de memoria, es activo y duradero, en la medida en que se reflexione sobre él. 
Una segunda iniciativa de trabajo a destacar es la de Sandra Patricia Rodríguez Ávila y Olga Marlene Sánchez Moncada, docentes en la ciudad de Bogotá de la Universidad Pedagógica Nacional, UPN, quienes desde el ejercicio de la docencia universitaria han venido abordando un tema en particular: el trabajo de la memoria en un país en guerra. Para estas investigadoras, producto de sus pesquisas y análisis se puede concluir que, hasta terminado el año 2009, no se habían difundido trabajos de tipo pedagógico que puedan involucrar explícitamente la historia reciente de Colombia en el currículo $y$, particularmente, el tema del conflicto interno. Tampoco se orientan trabajos de investigación y reflexión hacia el diseño de espacios de reelaboración de la memoria social desde las prácticas de enseñanza de la historia y de las ciencias sociales (Rodríguez y Sánchez, 2009 a). Para estas historiadoras y pedagogas no se encuentra en la agenda educativa una política de incorporación de la historia reciente o de la memoria del conflicto armado colombiano, ni como tema curricular ni como objeto de trabajo pedagógico.

Particularmente para Sandra Rodríguez, la discusión tiene como trasfondo el tema de "las políticas de la memoria", las cuales se constituyen a su vez en una categoría de análisis. Las políticas de la memoria consisten en un conjunto de estrategias diseñadas, desplegadas, reiteradas durante largos periodos de tiempo, y registradas para su actualización y transmisión, en la perspectiva de establecer, como proyecto social hegemónico de la población, un discurso en particular que no es más que la historia oficial. La lucha por las políticas de la memoria oficial se evidencia con la creación de la Academia de Historia y Antigüedades Patrias en 1902 y la Comisión Nacional del Centenario en 1907, instituciones que oficializan un discurso histórico y se oponen a la renovación de dicho discurso por parte de otros actores. Estas, y otra serie de instituciones oficiales, se constituyeron a lo largo de buena parte del siglo XX en instancias de saber histórico, difusores de estrategias editoriales planificadas, encargadas de la consolidación de un discurso oficial que dejó por fuera otras historias y otras memorias. Para Sandra Rodríguez (2009), detrás de este tipo de iniciativas basadas en la tradición de la autoridad monárquica, la autoridad de la cultura española, la autoridad moral de la Iglesia y la autoridad de las instituciones republicanas, se esconden buena parte de las políticas de la memoria oficial, las cuales inciden en la escuela por lo menos hasta 1984, cuando se da la reforma de las ciencias sociales escolares integradas.

Sandra Rodríguez y Marlene Sánchez, en su proceso de reflexión, han desarrollado un balance de la política educativa y las reformas con el fin de evidenciar el descuido de la historia reciente y del tema de la 
memoria en la escuela colombiana. En este sentido, las preocupaciones recientes se remontan al Decreto 1002 de 1984, que establece una propuesta integrada en ciencias sociales, la cual tuvo una incidencia directa en el tipo de trabajo histórico que se desarrollaba en la escuela. En esta reforma ni la historia reciente, ni el conflicto armado son considerados como parte del currículo, debido a que la dinámica de la reforma, en términos de integración, optó por planteamientos de carácter psicológico más que disciplinares. Luego, los Lineamientos curriculares en ciencias sociales de 1998, establecen una serie de propuestas didácticas que se inscriben en los avances propios de la psicología cognitiva, desarrollando planteamientos de la didáctica en las ciencias sociales, dejando una propuesta curricular de carácter más bien inductivo, flexible y abierto que depende de la iniciativa de los profesores.

No obstante la anterior dinámica, para estas investigadoras el tema de la memoria se convierte en el contexto colombiano en una demanda política, la cual no ha sido incorporada en el ámbito educativo, ni en proyectos de innovación e investigación pedagógica sobre la enseñanza de la historia reciente o del tiempo presente. De hecho, la iniciativa de Sandra y Marlene busca constituirse como una opción frente a las políticas de la memoria oficial. Dichas políticas son vistas como iniciativas de carácter público destinadas a difundir o consolidar una determinada interpretación de los acontecimientos del pasado, iniciativas que son de relevancia para un grupo social o político. Para el caso colombiano, estas investigadoras establecen tres tipos de memoria: la de los actores, la de las víctimas y la de la población civil. Frente a la anterior fuente, establecen una iniciativa de trabajo pedagógico en la escuela, el software Memoria, conflicto y relato (2009 a), que como material de apoyo de las prácticas educativas, permite reelaborar la memoria social del conflicto, articular la investigación disciplinar con las diversas iniciativas de las ciencias sociales escolares y visibilizarlas como problemática en el currículo. Uno de los objetivos fundamentales de este artefacto pedagógico es sensibilizar a los actores escolares frente a la temática como también la producción de nuevos relatos.

La intención del software Memoria, conflicto y relato, es constituirse en un material educativo para la enseñanza de las ciencias sociales en la educación media, el cual se ubica en la lógica de las nuevas tecnologías de la información y la comunicación 
escolar. Este material de apoyo en las prácticas educativas articula cuatro componentes analíticos que a la vez tienen un despliegue metodológico en el software: la reelaboración de la memoria social del conflicto armado colombiano; las prácticas narrativas de re-significación del pasado; la articulación de investigación disciplinar de las ciencias sociales con la investigación pedagógica y didáctica mediante la inclusión del conflicto armado colombiano como problemática curricular; y la incorporación crítica de las tecnologías de la información y la comunicación en la enseñanza de las ciencias sociales (Rodríguez y Sánchez, 2009 b). La propuesta mediante este artefacto tecnológico es articular la acción y la política tanto desde quienes investigan desde las ciencias sociales el tema de memoria, como de los colectivos que se benefician del resultado del trabajo académico. Otro de los componentes fundamentales de la propuesta pedagógica son los procesos narrativos, los cuales, más que como un género discursivo, son vistos como un mecanismo de reelaboración de la memoria y de la experiencia social del conflicto armado colombiano. La narrativa es un trabajo con la subjetividad en el que se involucran aspectos históricos del sujeto, de la sociedad y de la cultura que la constituye.

El software Memoria, conflicto y relato cuenta con un inventario de cinco tipos de fuentes: periodísticas, oficiales, actores armados, investigaciones académicas y expresiones culturales. Esta última es la más novedosa, pues encontramos obras plásticas, poesía, cuento, caricatura y humor político. En total hay 1.700 fuentes a consultar. En el artefacto educativo existen otro conjunto de categorías para ubicar fuentes de consulta: entrevista, estadística, cartografía, fotografía, testimonio y texto analítico. La incorporación crítica de las TIC tiene como objetivo fundamental generar procesos de subjetivación política y nuevas narrativas escolares que den cuenta de la relación traumática entre el conflicto y la memoria, desde un ángulo de carácter significativo y ejemplarizante.

En el ámbito educativo también pueden destacarse las iniciativas de Martha Cecilia Herrera, profesora del Doctorado en Educación Interinstitucional de la Universidad Pedagógica Nacional, Universidad Distrital de Bogotá y Universidad del Valle, quien ha venido promoviendo el tema de la memoria en la escuela en diversos espacios académicos como los seminarios de doctorado y maestría, en los cuales han participado varios de los investigadores pertenecientes a su grupo, espacios que se han constituido en un punto importante para el inicio de la reflexión en torno al tema. Para Martha Herrera, la memoria se constituye en un referente fundamental para dar cuenta de los procesos de socialización y subjetivación política en el interior de la escuela, teniendo 
como trasfondo el marco del conflicto político colombiano. La subjetivación política es comprendida como "un proceso a través del cual los individuos experiencian y se apropian, re-elaboran y re-significan los aprendizajes y prácticas relacionadas con la política y lo político, pautados por las instituciones, dando pie a la constitución de subjetividades políticas" (Herrera, 2009). La subjetivación se detiene más en los mecanismos de "agenciamiento", es decir, en cómo los individuos se constituyen a sí mismos en sujetos, tanto sociales como individuos, y se apropian e intervienen de manera diferente en el escenario de lo público.

Para Martha Herrera el tema de la memoria, de acuerdo a sus investigaciones realizadas en estos últimos años, ha vivido un proceso de activación política y de reconocimiento de una experiencia que nos configura como colectivo. La memoria se constituye en una materia para la interpretación del presente y de los proyectos de futuro, es una dimensión que debe buscar ser activada a favor de la consolidación de las memorias colectivas que permiten re-elaborar el actual conflicto político colombiano y contribuir a su solución.

\section{La experiencia situada con relación al tema de la memoria y la escuela}

En el ámbito escolar colombiano, las tensiones entre escuela, memoria y conflicto las ubicamos inicialmente desde finales de los años noventa, cuando se le dio peso a la oralidad y las fuentes orales, reivindicando en algunos momentos la voz del sujeto escolar, el niño escolarizado. En un país inmerso en una lógica de violencia endémica permanente, la "memoria del horror" y la tragedia colectiva no parecía sensibilizar aún las posibles temáticas que abordaba el docente de ciencias sociales en la escuela. La violencia se difuminó como temática en los años ochenta al regionalizarse, al periodizarse y al caracterizarse como un fenómeno más rural que urbano, por parte de los investigadores de la violencia de ese entonces. Otros temas, como el desarraigo, el desplazamiento y la inclusión social, ganaban mayor relevancia para algunos investigadores sociales externos a la escuela, y no propiamente para el docente investigador.

En la escuela colombiana, el tema de la memoria y el olvido pasó primero por trabajar de manera directa temáticas afines como las narrativas infantiles y juveniles, la oralidad y la historia 
oral, la historia local y su relación con la historia regional y nacional. En este sentido, fueron importantes los esfuerzos de Renán Vega Cantor, quien a finales de los años noventa en la Universidad Pedagógica Nacional, acercó a un número importante de docentes en ejercicio con el fin de trabajar la fuente oral con los estudiantes y su relación con la historia local. En el primer texto, ¡Déjenos hablar! (Vega y Castaño, 1999), además de un ejercicio de historias locales, descuella un importante capítulo en torno a la disciplina histórica, la historia oral y la enseñanza de la historia, en el que se establecen pistas para abordar el tema de la oralidad en la escuela. En su propuesta deja claro la importancia que tiene la fuente oral como una fuente viva, reivindicando la subjetividad tanto en el conocimiento como en las manifestaciones culturales. Mediante la oralidad, la intención del investigador es la de reconstruir atmósferas, aspecto que es tan importante e inclusive muchas veces más significativo que establecer la precisión cronológica de un acontecimiento, ya que implica las pulsaciones de la mentalidad colectiva, las dimensiones de la cotidianidad, constituyéndose en una expresión de la cultura. En este texto se logran sistematizar al menos once experiencias docentes, en la que si bien el tema central es la historia oral, la relación que en el proyecto investigativo se establece con la memoria termina siendo fundamental.
En un segundo libro, Historia: conocimiento y enseñanza. La cultura popular y la historia oral en el medio escolar, Renán Vega aborda elementos de gran envergadura para el maestro en ejercicio, como la tradición oral, la historia de vida, la historia oral y las tipologías de historia oral que se podrían trabajar en la escuela. Inspirado en autores de la oralidad como Esteban Monsoyini (1990) y Jaen Vansina (1982), deja sentadas las bases para pensar el peso que gana la oralidad en las ciencias sociales escolares y reivindica la altura que debe tener la oralidad en las aulas de clase. La historia oral hace parte de la palabra viva, de las cosmovisiones, creencias y luchas de los colectivos sociales. En su abordaje el docente investigador debe tener en cuenta el "marco social" y la tradición donde surge la fuente oral, como también la función social que desempeña. También el investigador debe valorar el "marco mental" de la tradición con el fin de comprender lo científico de una sociedad que la diferencia de otra para encontrar sus modelos de acción, ideales y valores (Vega, 1999). Cuando nos acercamos a este tipo de propuestas nos encontramos con una historia en la que cuenta la subjetividad, relacionada con la fuerza del recuerdo, la memoria y la manera como el sujeto reconstruye su experiencia. En el marco de sistematización de la experiencia y escritura de este segundo libro Renán Vega, desde la Universidad Pedagógica 
Nacional-UPN, en coordinación con el IDEP, lidera una serie de cursos de profesionalización docente en el que un importante número de docentes en ejercicio se acercan al tema de las fuentes orales escolares y, por este camino, a la tensión del recuerdo y la memoria que este ejercicio conlleva cuando se aplica como dispositivo de trabajo pedagógico en el interior de la escuela.

Fruto de este esfuerzo son las investigaciones de Fabio Castro Bueno, docente de la Secretaria de Educación-SED, quien participa en la conformación, para el año 2005, del Centro de Memoria en Educación y Pedagogía del IDEP, en la ciudad de Bogotá. El interés de este centro es conservar la memoria de la práctica pedagógica para evidenciar las múltiples formas como esta se genera, aportar a la constitución de su discurso y a la constitución de una identidad en particular dentro del colectivo docente de la ciudad. Esta iniciativa establece una relación directa entre la historia, la memoria, las tradiciones y las fuentes orales. En una investigación financiada por el IDEP, en la que indaga la memoria de 47 docentes en ejercicio que trabajan en sus didácticas la fuente oral y la oralidad, desde los métodos propios de enfoque cualitativo, se logra establecer que existen unos niveles de memoria de carácter individual, colectiva e histórica, que puede potenciar el docente en ejercicio. Soportado en Josep Fontana, desarrolla la categoría de "memoria personal", vértice de su discusión y metodología para dar cuenta de "un complejo sistema de relaciones que tiene un papel fundamental en la formación de conciencia y que la conciencia se vale de la memoria para poner en juego las experiencias previas y para diseñar un escenario al cual pueda incorporar los elementos nuevos" (Castro, 2004). La memoria colectiva acompañada de las nuevas tecnologías simplemente se ha transformado, constituyéndose en un nuevo vínculo y soporte de la memoria contemporánea, siendo inherente al hombre conservar su memoria de acuerdo a ciertas posibilidades técnicas de registro y representación: la pintura, la escritura y las nuevas tecnologías. Para Castro, el docente en ejercicio debe tener en cuenta que existen memorias plurales que se refuerzan y complementan o que se oponen y contradicen. El educador debe conocer y manejar esta situación, ya que esta discusión hace parte de las contradicciones políticas e ideológicas que se libran tanto en la escuela como fuera de ella. La recuperación de la memoria histórica tiene como fuente fundamental en su propuesta la suma de "memorias personales", 
la fuente oral y la oralidad, la cual se caracteriza por ser una expresión de palabras habladas, expresión de pensamientos, percepciones, imágenes, recuerdos hablados, relatados o narrados, que han sido provocados y registrados en una libreta de apuntes o diario. La oralidad y su relación con la memoria se deben valorar como construcciones de significado y simbólicas del sujeto, que como expresión de una relación subjetiva garantizan un alto grado objetividad en las metodologías que se proponen para la escuela por parte de un grupo importante de docentes en ejercicio.

Por otro lado, en la Universidad Distrital Francisco José de Caldas de Bogotá, bajo la lógica inicial de este tipo de esfuerzos, si bien no se hicieron investigaciones a finales de los años noventa en torno a la relación que existe entre la memoria y la escuela, sí se acumuló un esfuerzo importante en torno al papel de la narrativa infantil como una herramienta fundamental por medio de la cual se organiza la experiencia y se materializa la memoria. En este sentido, las investigaciones iniciales se manejaron desde la perspectiva de Jerome Brunner, para quien el pensamiento narrativo en el niño, de la misma manera que un relato, incorpora la realidad social y cobra sentido a partir de los agentes, personajes, ambientes, escenarios, intencionalidad, relaciones y acciones (Gómez y Ramírez, 2000). En consecuencia, mediante el pensa- miento narrativo, la modalidad narrativa:

El sujeto logra atribuirle un significado a su propia experiencia, ya que la narración media entre el mundo canónico de la cultura y el mundo más idiosincrásico de las creencias, los deseos y las esperanzas, hace que lo excepcional sea comprensible. La modalidad narrativa del pensamiento se preocupa más por la verosimilitud que por la verdad, sus mecanismos tienen que ver más con lo simbólico, analógico y la construcción intersubjetiva de lo social que con la equilibración de estructuras lógicas (Gómez Esteban, 2002, p. 39).

Desde la perspectiva de J. Brunner, impulsada por Jairo Gómez Esteban en la Universidad Distrital, se lograron sacar adelante una serie de trabajos en campo que articularon el tema del pensamiento narrativo del niño con otras dos categorías fundamentales en el análisis, experiencia y memoria, en contextos escolares.

Años después, el mismo Jairo Gómez termina de madurar sus hipótesis de trabajo, al relacionar las categorías de narrativa y memoria en un pequeño ensayo en el que desarrolla una propuesta para trabajar el tema en la escuela. Para este investigador, 
la memoria se construye sobre la narrativa del sujeto, la cual va moldeando una singularidad que se reconoce en la posibilidad de decir-se, de nombrar-se y expresar-se. Por medio de la narrativa y la memoria del sujeto se puede avanzar en los procesos de subjetivación política liderados por la escuela. Para Gómez, la investigación social contemporánea "le apuesta fundamentalmente a la producción de conceptos y categorías fronterizas, en los bordes y los límites tanto de las disciplinas científicas como de los saberes no científicos, artísticos, estéticos, culturales y los de sentido común" (Gómez, 2009). Apoyado en J. Brunner (2002 y 2006), Jairo Gómez manifiesta que el pensamiento narrativo, de la misma manera que un relato, incorpora la realidad social y cobra sentido a partir de agentes, actores, ambientes, escenarios, intencionalidades, relaciones y acciones. Mediante la narrativa el sujeto logra atribuirle un significado a su propia experiencia, ya que la narración media entre el mundo canónico de la cultura y el mundo idiosincrásico de las experiencias, los deseos y las esperanzas. El pensamiento narrativo está mediado por los relatos circundantes en el imaginario colectivo, por la nomenclatura que un grupo social ha construido para darle sentido y significado a su experiencia y a su vida. De acuerdo a la propuesta de Jairo Gómez, apoyada en varios autores, existen cinco criterios para que una narración sea inteligible: establecer un punto final apreciado, seleccionar los acontecimientos relevantes para el punto final, la ordenación de los acontecimientos, la estabilidad de identidad en el relato, vinculaciones causales e intencionalidad de los actores y, por último, el signo de demarcación en la narrativa, pues los relatos emplean un principio y un final.

Bajo la perspectiva de los principios del pensamiento narrativo y la experiencia, los investigadores Absalón Jiménez Becerra y Raúl Infante Acevedo abordan el tema de las narrativas infantiles en Bogotá, con un grupo de niños en situación de exclusión social, ubicados en un rango de edad entre los 8 y 14 años, cuyo interés es rescatar su experiencia y noción de ciudad (Jiménez e Infante, 2008). La investigación, que se realizó bajo un enfoque cualitativo, buscó inspeccionar el pensamiento narrativo del niño desde la perspectiva de J. Brunner, echando mano de un número importante de iniciativas metodológicas que permitieron al niño reconstruir su historia de vida y experiencia de ciudad de una manera fluida. La relación entre experiencia, pensamiento narrativo y memoria es fácilmente palpable a lo largo 
de la lectura de la investigación, en la cual uno de los principales compromisos éticos fue el de incorporar la voz de los sujetos, en este caso la voz de los niños que participaron en la experiencia investigativa.

Por su parte, Absalón Jiménez B., como profesor de la Universidad Distrital, una vez finalizada la anterior experiencia, hila otra serie de discusiones con el fin de seguir trabajando el tema con los docentes en ejercicio y con los estudiantes de licenciatura y maestría. Para tal fin escribe el artículo: "Memoria, historia y escuela" (2008), con el interés de dar cuenta de cómo el tema de la memoria para la escuela y los docentes se presenta con un doble perfil de preocupación: uno de carácter pedagógico y otro de carácter político. En lo que respecta al carácter pedagógico del problema, la memoria atraviesa el tema de las nociones sociales de tiempo y de espacio, por medio de las cuales el sujeto individual organiza su experiencia. La memoria como expresión de experiencia se ancla en este tipo de nociones, las cuales el docente en ejercicio debe valorar y debe tener las herramientas para trabajarlas con los estudiantes. En lo pedagógico, el interés de Jiménez $\mathrm{B}$. es fortalecer una propuesta de carácter conceptual y metodológico, con el fin de que el docente en ejercicio tenga una serie herramientas para la intervención en la temática de la memoria, tensionándose así la memoria oficial, particularmente la historia oficial y las ciencias sociales escolares.

En los referentes conceptuales, la propuesta de Jiménez $B$. toma a Paul Ricoeur para dar cuenta de la memoria como un ejercicio de evocación de lo ausente y de representación del pasado, que compromete una discusión de carácter epistemológico, hermenéutico y metodológico. A lo largo de su propuesta busca dar cuenta del peso que tiene la oralidad como hilo conductor entre la memoria y la historia, y particularmente entre la memoria y la escuela. En este sentido, es importante que el docente en ejercicio tenga en cuenta las diferencias entre memoria e historia. La memoria se mueve en el campo de la subjetividad, en la verosimilitud, en el carácter instaurador, desordenado y palpitante de la narrativa del sujeto. La historia por su parte se mueve en el campo de la objetividad, la exactitud, el ejercicio racional, en un papel aclarador, en el orden y en la lógica de un discurso disciplinar, y en la lógica propia de una ciencia. Para Jiménez B. es importante que el docente en ejercicio establezca unos niveles de la memoria: memoria individual, memoria colectiva y memoria histórica, tomados de la mano de autores como Maurice Halbwachs (2004) y Darío Betancourt (2004). Desde la perspectiva conceptual de "los marcos de la memoria", les da a conocer a los maestros en ejercicio el papel del recuerdo como un elemento de tensión entre la memoria y 
el olvido y facilita algunos elementos para trabajar la narrativa y el pensamiento narrativo en el sujeto.

En lo que respecta al carácter político del tema de la memoria en la escuela, para este investigador el docente en ejercicio, al igual que el historiador, representa en la actualidad a uno de los principales "guardianes" de la memoria oficial e institucional. Los primeros por medio de su labor investigativa, en un alto porcentaje han participado de la oficialización de un relato alegórico, heroico y decimonónico constituido por las élites. Y los segundos de manera implícita desde su práctica oficializan el relato cuando sin mayor reflexión participan de la celebración en el contexto escolar de fiestas políticas nacionales como la del 20 de julio, 6 y 7 de agosto, 12 de octubre, etc.; o cuando desde su práctica se ciñen a un currículo con unos contenidos que simplemente expresan unas políticas de la memoria oficial, que deja por fuera a la memoria de esos otros. En buena medida la escuela, por medio de esas otras memorias, puede tensionar a la historia oficial, ejercicio mediante el cual una "memoria ejemplarizante", trabajada por Todorov (1995) en su libro Los abusos de la memoria, se constituye en una posibilidad de trabajo con el fin de re-construir esa otra memoria y esa otra historia.

Para el año 2007 encontramos la experiencia patrocinada por el IDEP y liderada por Lola Cendales: "Reconstrucción de la memoria pedagógica", la cual se realizó en varias instituciones escolares de Bogotá. En esta se lograron sistematizar once experiencias que se enmarcaron en varios temas, tales como: historia de la institución escolar, recuperación de las prácticas pedagógicas y relaciones entre escuela y comunidad. Este último fue un proceso de formación y acompañamiento a los docentes en ejercicio con el objetivo fundamental de cualificar su formación en la escuela. En esta iniciativa se destacó la investigación de Juan Carlos Amador y Pilar Albadán, quienes dan cuenta de la recuperación de la memoria educativa y pedagógica de dos instituciones escolares del suroccidente de Bogotá, entre 1970 y 2007. El tema es abordado desde las subjetividades docentes, espacio en el que la narración y la experiencia se configuran mediante la potencia del recuerdo. En este sentido, la experiencia de sí como exposición de subjetivación en la cual el ver-se, el narrar-se, el expresar-se, el juzgar-se y el dominar-se, representan una posibilidad de reactualizar la memoria y la identidad del docente en ejercicio. Para estos docentes investigadores la 
recuperación de la memoria desde la voz de los actores es condición y oportunidad, no solo para develar otras versiones de lo ocurrido en el tiempo, sino para delinear caminos posibles, trayectorias de vidas personales y colectivas.

Los referentes conceptuales de la propuestas se mueven en la perspectiva de subjetivación que manejan autores como Michel Foucault (1996) y Jorge Larrosa (1992), quienes hablan desde las tecnologías del yo como una posibilidad para dar cuenta del gobierno de sí y la autoconstrucción del sujeto. La categoría de memoria atraviesa la propuesta teniendo en cuenta la perspectiva de Paul Ricoeur (2000), quien observa la memoria como la evocación de lo ausente, y también la de experiencia y narración de Walter Benjamin. La escuela, desde la perspectiva de Maurice Halbwachs (2004), se convierte en el principal "marco de la memoria" institucional para recuperar el recuerdo, en donde la rememoración, experiencia y constitución de sujetos se convierten en referentes de la fabricación de subjetividades que caracterizan la singularidad y la contingencia de un sujeto proclive a la dominación. Desde una perspectiva inspirada en Foucault, los investigadores promueven nuevas formas de subjetividad por medio del rechazo de un tipo de individualidad impuesto durante siglos por las disciplinas tradicionales. La memoria es asumida como objeto y mediación para mirarse a sí mismo, desglosar la propia existencia en el tiempo y explicar lo ausente, es un mecanismo para avanzar en la investigación desde una perspectiva interdisciplinar en el interior de la escuela (Amador y Albadán, 2009). Las dimensiones de la experiencia de sí, vistas como expresión de subjetivación: el ver-se, narrar-se, expresar-se, juzgar-se y dominar-se, sirvieron para recoger una multiplicidad de información de la voz de los actores. Estas categorías facilitaron los elementos para la construcción de una matriz de análisis, en el que se ubican las tecnologías disciplinares y pedagógicas. Lo que se logró comprobar a lo largo de la investigación es que la escuela cumple una función de operador óptico que le permite a los docentes auto observarse y reconstruir su experiencia institucional en compañía de la comunidad educativa.

Por su parte, Andrés Fernando Castiblanco Roldán, entonces docente de la Secretaría de Educación del Distrito-SED, desarrolla hacia el año 2006 una iniciativa de trabajo en torno a la memoria vista con posibilidades metodológicas al interior del mundo escolar. De este profesor podemos destacar dos experiencias: la primera tiene que ver con el tema de la memoria y espacio lúdico, en la que por medio de una serie de iniciativas eminentemente escolares establece una relación entre el espacio, los lugares y la memoria. Desde su perspectiva existen dos formas de espacio social memorables: uno institucional, 
conmemorativo, alusivo y oficial que genera escenarios y empresas; $y$ otro discursivo y simbólico, permanente en los lugares, representaciones y propósitos. Uno de sus principales referentes conceptuales es Marc Augé (1994 y 1998), para quien el espacio como lugar practicado, fruto del movimiento y los cruces entre elementos que son dinámicos, termina conformando relatos de espacio. También hace referencia en su propuesta a Maurice Halbwachs, quien por medio de los "marcos de la memoria" hace posible que la memoria pueda consolidarse y preservarse en un grupo, en el devenir temporal con grandes referentes institucionales y sociales como son: la familia, la religión, el lenguaje y el espacio. La memoria individual y la memoria colectiva se ponen en juego como representación del pasado, constituyendo memoria con relación a un espacio físico. En su propuesta de intervención escolar establece una relación directa entre el espacio físico vivido por el sujeto como parte del escenario indómito y su relación con la lúdica, el circo, la fiesta y el carnaval y su relación con el juego. Para el profesor Castiblanco, el escenario se presenta como materialización del deseo lúdico y los espacios van definiendo y generando nuevas formas y sentidos a las acciones y lecturas que se establecen de la realidad.

En una segunda iniciativa, el trabajo desarrollado por Castiblanco involucró de nuevo a los estudiantes de su institución escolar, lo que conllevó a tomar conciencia sobre la memoria colectiva y que requirió el retorno al hogar, volver al diálogo con los padres o tutores para hablar del pasado común. Este docente aborda el concepto de memoria como parte de un "marco social" investido por la sociología de Halbwachs, la cual sirve como ruta conceptual para desarrollar el trabajo. Los "marcos de la memoria" permiten reconstruir recuerdos sobre una serie de convergencias de acontecimientos y lugares comunes. No obstante, la memoria también se halla revestida por el silencio al cual el investigador le debe dar una relevancia importante en el momento de su intervención. La escuela es una posibilidad de constitución de archivo en la que "los portafolios de memoria", encuestas, grupos focales y análisis de la imagen, vistos como documento, se convierten en una posibilidad metodológica de trabajo. En su propuesta los "portafolios de la memoria": la imagen, el álbum fotográfico, la autobiografía y la transformación del espacio, se constituyen en una posibilidad importante de trabajo con los niños y jóvenes en la escuela (Castiblanco, 2009). 
Para este docente, la escuela y el aula son el ágora donde confluyen diferentes clases de relatos, desde la producción del discurso académico hasta las historias de la cotidianidad respecto del fin de semana. La recopilación de los registros y anécdotas fueron divididos en tres categorías: lo lúdico, lo social y lo escolar. En lo social, se abordó la memoria de la violencia en Colombia, la memoria de los movimientos sociales y las memorias religiosas y emergentes. Dentro de la memoria de la violencia, la narrativa de los estudiantes, acompañada con la de sus padres, le dio relevancia a acontecimientos como la toma al Palacio de Justicia por parte del M-19 en 1985 y la muerte de Pablo Escobar en 1993. Dentro de la memoria de los movimientos sociales, el Paro Cívico de 1977 fue rememorado por los padres de familia y los estudiantes. En las memorias religiosas y emergentes la visita del papa Juan Pablo II en 1986 fue un acontecimiento rememorado por medio de esta metodología. La anterior iniciativa es importante porque la experiencia cotidiana se entremezcla con la experiencia social y colectiva, constituyéndose un tipo de memoria en la que participan los sujetos escolares: docentes, estudiantes y padres de familia. Este puñado de anécdotas y suma de recuerdos se constituye en un elemento importante para reconstruir la memoria de una nación y la constitución de una identidad colectiva, muchas veces sufrida $y$ excluida, que la escuela puede promover.

Otra investigación que es importante destacar es la encabezada por Orlando Silva Briceño, profesor de la Universidad Distrital, quien por medio de una propuesta investigativa de carácter genealógico indaga la memoria del periodo de la Violencia en Colombia, el cual es vivido a mediados del siglo XX. El tema es trabajado en seis instituciones educativas, evidenciando la situación de esta temática en el marco del debate actual entre la memoria oficial y otras memorias. Su investigación reconoce a la escuela como un dispositivo de poder en el que juegan un papel fundamental los sujetos y los saberes. Desde su perspectiva se da cuenta de un alto grado de subordinación de la memoria que puede ser vista como parte de esos "saberes sometidos" que se insubordinan frente al currículo oficial de acuerdo a ciertas condiciones de posibilidad recientes. Las ciencias sociales, su enseñanza y la relación que puede establecer con la memoria están compuestas por tensiones y tendencias inmersas en las lógicas de saber y poder. La memoria surge así como un saber emergente, logrando una expansión en el campo académico y político, expresada en distintas manifestaciones ligadas fundamentalmente a los sucesos conflictivos que han caracterizado el país (Silva, 2010).

La disciplina histórica, al funcionar como dispositivo de disciplinamiento de los discursos, configura 
esquemas de oposición para determinar lo que sería verdadero o falso de los discursos sobre el pasado, que asociado a cierto tipo de narrativas constituye la historia oficial. En la escuela contemporánea colombiana encontramos unas tradiciones fuertes y unas rupturas débiles en las prácticas de enseñanza en las ciencias sociales, en las que podemos confundir los ejercicios de la memoria con la memorización de información y lógicas tradicionales de evaluación de las actividades. No obstante, los ejercicios recientes de la memoria y su uso con relación a la historia nacional en la escuela, son utilizados como una herramienta ejemplarizante para la no repetición de acontecimientos dolorosos, relación que aún es muy tenue, pero que se está materializando en determinadas iniciativas didácticas y pedagógicas. La escuela puede ser utilizada como un "marco de la memoria" y arista de "agenciamiento" para su aplicabilidad. En este sentido, se hace necesario exponer las características de las memorias de la primera violencia agenciadas al interior de la escuela, establecer los documentos que sustentan dichas narrativas, dilucidar los marcos de regulación social que las reactualizan y, por último, determinar la función existente entre otros y las características de la institución escolar (Silva, 2010, p. 203).

\section{Conclusiones}

Como puede verse, uno de nuestros intereses en el presente estado del arte fue poner en orden una bibliografía y una serie de experiencias iniciales realizadas en la escuela con relación al tema de la memoria. En lo que respecta a nuestra primera preocupación, la de contextualizar el tema dando cuenta del momento en que la memoria como categoría logra cierta relevancia en la escuela, podemos anotar el año de 1997, cuando el tema entra por la puerta de la historia oral, la oralidad y la fuente oral, teniendo como principales apoyos institucionales la Universidad Pedagógica Nacional y el IDEP. Otro referente es el año 2000, cuando desde la Universidad Distrital de Bogotá se logran las primeras publicaciones que dan cuenta de lo narrativo como iniciativa investigativa en la escuela, particularmente el tema del pensamiento narrativo y la representación histórica. Las anteriores iniciativas en su momento daban cuenta de manera implícita de la preocupación por el carácter subjetivo en torno al tema del sujeto y su abordaje. También se lograba establecer la cercanía de una serie de temas como lo era la oralidad, la 
fuente oral, la narrativa y su relación con la experiencia y la memoria. La categoría de memoria de manera explícita se logra ubicar en una serie de iniciativas del 2005 a la fecha, de manera inicial en el Colectivo de historia oral, conformado en su mayoría por profesores del Distrito, y hacia 2006 cuando Olga Lucía Zuluaga Garcés deja las bases de la categoría: memoria activa del saber pedagógico, mediante la cual tenía como intención organizar una serie de iniciativas de trabajo silenciadas y sometidas en la escuela a procesos de "invisibilización" por parte de cierto tipo de discurso oficial. Luego, en el año 2007, ubicamos la iniciativa del IDEP, liderada de manera particular por la investigadora Lola Cendales, quien por medio de una serie de metodologías de carácter cualitativo logra en compañía de un equipo de trabajo conformado por docentes en ejercicio, la reconstrucción de la memoria pedagógica de varias instituciones del Distrito Capital. Para esta misma época también se ubican una serie de iniciativas en el ámbito universitario por parte de destacados docentes e investigadores que hacen parte de las facultades de educación de las universidades públicas de la ciudad de Bogotá.

Por lo demás, podemos anotar que las iniciativas de la memoria en la escuela como temática y dispositivo pedagógico de intervención, se institucionalizan en Colombia de manera paulatina en la primera década del siglo XXI, producto del ejercicio mismo de la práctica docente y en momentos en que se da un proceso de "desmovilización" por parte de actores armados caracterizados como de derecha, lo que lleva a la sociedad colombiana a hablar, desde el año 2005, de una Ley de verdad, justicia y reparación de víctimas. En este sentido, la memoria de los individuos y los colectivos juega un papel fundamental para la materialización del primer objetivo de la ley, como lo es la verdad; para que haya justicia es fundamental la reconstrucción de la verdad histórica al menos de las violencias recientes que han afectado al país, lo que demanda un compromiso institucional de la escuela para abordar el tema. Otro elemento que está en el trasfondo de varias de las iniciativas balanceadas es la situación de una memoria irresuelta, de una memoria rota, con relación a los conflictos recientes, empezando por el genocidio y desaparición sistemática de un partido político, la Unión Patriótica, a finales de los años ochenta; y las masacres y desapariciones forzadas de los paramilitares de los años noventa y las secuelas del conflicto interno con las FARC, cuyos principales afectados son las comunidades regionales. Como investigadores creemos, producto del presente balance, que el tema de la memoria en la escuela se constituye en una demanda política y pedagógica que de manera paulatina se seguirá visibilizando en las iniciativas de trabajo de 
los docentes en ejercicio, como dispositivo de intervención e inclusive como temática en el currículo.

En el ámbito de lo conceptual se puede decir que la memoria no es un objeto acabado con contornos claramente trazados, sino que cuenta con una perspectiva interdisciplinar para su abordaje y reflexión. Como se pudo evidenciar, la memoria comparte fronteras con conceptos vecinos como el de oralidad, fuente oral, experiencia, narrativa y pensamiento narrativo. También producto del balance podemos dar cuenta de una serie de autores que se constituyen en referentes fundamentales para desarrollar iniciativas de carácter investigativo en la escuela: en primer lugar autores como Paul Ricoeur (2001), para quien la memoria representa un ejercicio de evocación del pasado y representación de lo ausente; Maurice Halbwachs (2004), con "los marcos de la memoria", particularmente los marcos institucionales de la memoria en los que se ubica la escuela y los niveles interrelacionados de memoria individual y colectiva; Tzvetan Todorov (1995), con relación a los abusos de la memoria y la posibilidad de trabajar "la memoria ejemplarizante" con base en experiencias dolorosas por las que ha atravesado nuestra sociedad; y Elizabeth Jelin (2002 y 2004), quien estableció la importancia del tema de las políticas de la memoria oficial, las cuales tienen como principal escenario de lucha y tensión la escuela. Desde la perspectiva de la subjetivación del sujeto, en la que se establecen unas condiciones de posibilidad para que este se vea, se narre, se exprese, se juzgue y se domine, encontramos como referentes obligados a Foucault (1996) y Larrosa (1992). Ya en el ámbito nacional observamos una serie de iniciativas de trabajo que han decantado, por medio de su relación con la memoria, la categoría de "subjetivación política" en la que ubicamos a Martha Cecilia Herrera (2009), Jairo Gómez Esteban (2009) y Sandra Rodríguez (2009).

En lo que respecta a las metodologías de la memoria en contextos escolares, podemos manifestar que buena parte de las iniciativas se caracterizaron por un elemento en común, el carácter vinculante de los sujetos en las iniciativas de trabajo. La memoria conllevó un acercamiento a las narrativas del sujeto, a sus diversos grados de experiencia y a la materialización de relatos como expresión de memoria desde una perspectiva subjetiva, en la que lo cualitativo y sus métodos se constituyen en una posibilidad de abordaje. En esta perspectiva ubicamos las iniciativas de Renán 
Vega y Fabio Castro con respecto a la oralidad, la fuente oral y la historia oral, en la que la experiencia de los sujetos investigados, es decir, la relación sujeto-sujeto, está mediada por una serie de ejercicios narrativos motivados por las inquietudes del investigador. También se ubican otra serie de estrategias que parten del carácter vinculante del docente mismo, en las que la perspectiva de la subjetivación establece la posibilidad de ver-se, narrar-se, expresarse, juzgar-se y dominar-se, iniciativas en la que se ubican la mirada de Juan Carlos Amador y Pilar Albadán y la del mismo Jairo Gómez Esteban. Como tercera metodología importante a destacar tenemos la de quienes desde los "marcos de la memoria" han utilizado la escuela y la ciudad como contextos institucionales y culturales para desarrollar diversas iniciativas de trabajo de tensión entre la memoria y la escuela, que comprometen a la familia y la comunidad. Se destacan las metodologías de trabajo aplicadas por Andrés Castiblanco. También se destaca la iniciativa de Sandra Rodríguez Ávila y Marlene Sánchez, quienes por medio del uso crítico de la nuevas tecnologías de la información y la comunicación buscan acercar a los estudiantes a las fuentes, con el objetivo de generar unas condiciones particulares en las que el conflicto armado y político se visibilice en la escuela y se logren materializar nuevos relatos que valoren esas otras memorias excluidas de la escuela. En general, la memoria vista como dispositivo de intervención pedagógico se puede rescatar desde la misma cotidianidad de los sujetos escolares, ser abordada desde diversas metodologías, trabajada desde diversas fuentes, ambientada con diversas ayudas didácticas $y$, en general, acompañada de experiencias propias del sujeto docente. Experiencias que se constituyen de manera implícita en una determinada concepción de práctica y saber pedagógico, tanto en el ámbito escolar como a nivel universitario.

La lucha por la memoria oficial se evidencia en la escuela, particularmente en asignaturas como la Historia y las ciencias sociales escolares, que al seguir un lineamiento oficial se inscriben en las políticas de la historia oficial del Estado. No obstante, en la actualidad algunos maestros, en la escuela, han logrado tensionar los ángulos de la memoria y la historia oficial recurriendo a experiencias significativas y de innovación de trabajo pedagógico donde el tema del conflicto social, político y armado, vivido tanto en el escenario rural como el urbano, se ha convertido en una posibilidad temática de trabajo en el aula. Parafraseando a alguna de las investigadoras, la memoria como proceso subjetivo se encuentra anclada en experiencias y en marcas simbólicas y materiales que la escuela debe valorar.

Otra conclusión que nos ha dejado el presente balance es el de "des-fatalizar" la memoria y asumirla como 
objeto y mediación para mirarnos a nosotros mismos como proyecto colectivo, como proyecto de nación. La memoria vista como parte de un saber emergente y como parte de una demanda y preocupación política y pedagógica, puede ser re-construida bajo la lógica de la suma de un puñado de anécdotas y suma de recuerdos que constituyen un elemento importante para la constitución de una identidad colectiva, muchas veces sufrida y excluida, que la escuela no puede desconocer.

\section{Referencias}

Augé, M. (1994). Los no lugares, espacios de lo indómito. Barcelona: Gedisa.

Augé, M. (1998). Las formas del olvido. Barcelona: Gedisa.

Amador, J. C. y Albadán, P. (2007). Memorias y subjetividades en la escuela oficial: experiencias de sí y políticas educativas, 1970- 2007. En: Lola Cendales. Reconstrucción de la memoria pedagógica. Bogotá, D.C.: IDEP.

Betancourt, D. (2004). Memoria individual, memoria colectiva y memoria histórica. En: Jiménez B. A. y Torres, A. La práctica investigativa en ciencias sociales. Bogotá, D.C.: Universidad Pedagógica Nacional.

Brunner, J. (2000). Actos de significado. Más allá de la revolución cognitiva. Madrid: Alianza.

Brunner, J. (2002). Acción, pensamiento y leguaje. Madrid: Alianza.

Castiblanco Roldán, A. (2009). De las anécdotas a la mega narración de la memoria: historia y espacios en la escuela. En: Jiménez, A. y Guerra García, F. (Compiladores). Las luchas por la memoria. Bogotá, D.C.: Ipazud, Universidad Distrital.

Castiblanco Roldán, A. (2008). Memoria y espacio lúdico: goce, juego, lugares y anónimos. En: Serna Dimas, A. Memoria y conflicto. Cátedra democracia y ciudadanía. Bogotá, D.C.: U. Distrital, Ipazud.

Castro Bueno, F. (2005). Caracterización pedagógica de prácticas docentes como aporte a la constitución del Archivo Pedagógico en Bogotá, Archivo IDEP, (Inédito).

Castro Bueno, F. Historia oral: historia de vida e historia barrial. Bogotá D.C., Colectivo de historia oral, 2004.

Dussel, I., Finocchio, S. y Gojma, S. (2003). Haciendo memoria en el país de nunca más. Buenos Aires: Eudeba. 
Foucault, M. (1996). Las tecnologías del yo. Paidós: Buenos Aires.

Foucault, M. (1976). Historia de la sexualidad. La voluntad de saber. Tomo I. México: Siglo XXI.

Gómez Esteban, J. y Ramírez, P. (2000). Conocimiento social y enseñanza de la historia Bogotá D.C.: UD-CIDC.

Gómez Esteban, J. y Ramírez, P. (2000). La representación infantil del mundo social en el aula de clase: las nociones sociales. Bogotá D.C.: UD-CIDC.

Gómez Esteban, J. H. (2002). La construcción del conocimiento social en la escuela. Bogotá D.C.: UD-CIDC.

Gómez Esteban, J. (2009). Voces que quieren hacerse oír. Narrativas de la memoria y el olvido en la escuela. En Jiménez B., A. Las luchas por la memoria. Bogotá, D.C.: Ipazud, Universidad Distrital.

Herrera, M. (2009). Políticas de la memoria como forma de socialización y de subjetivación política: un análisis histórico sobre el tiempo presente. En: Jiménez B., A. Las luchas por la memoria. Bogotá, D.C.: Ipazud, Universidad Distrital.

Halbwachs, M. (2004). Memoria colectiva. Zaragoza: Prensa Universitaria.

Jelin, E. (2002). Los trabajo de la memoria, Buenos Aires: Siglo XXI.

Jelin, E. y Lorenz F. G. (2004). Educación y memoria. La escuela elabora el pasado. Buenos Aires, Siglo XXI.

Jiménez Becerra, A. (2004). El estado del arte en la investigación en las ciencias. En: La práctica investigativa en ciencias sociales. Bogotá, D.C.: Universidad Pedagógica Nacional.

Jiménez Becerra, A. (2008), Memoria, historia y escuela. En: Serna Dimas, A. Memoria y conflicto. Cátedra democracia y ciudadanía. Bogotá, D.C.: U. Distrital, Ipazud.

Jiménez Becerra, A. y Guerra García, F. Compiladores (2009) Las luchas por la memoria. Bogotá, D.C., Ipazud, Universidad Distrital.

Jiménez Becerra, A. e Infante Acevedo, R. (2008), Infancia y ciudad en Bogotá. Una mirada desde las narrativas populares urbanas. Bogotá, D.C.: U. Distrital de Bogotá- CIDC.

Larrosa, J. (1992). Tecnologías del yo y educación. En: Escuela, poder y subjetivación. Madrid: Puiqueta.

Monsoyoni, E. (1990). La oralidad. En: Oralidad. Anuario para el rescate de la oralidad en América Latina y el Caribe No 2. La Habana.

Ricoeur, P. (2000). La memoria, la historia, el olvido. México: Fondo de Cultura Económica.

Rodríguez Ávila, S. P. (2009). Producción, difusión y consolidación de la memoria oficial en Colombia En: Jiménez Becerra, 

sidad Distrital.

Rodríguez, S. P. y Sánchez, O. (2009 a). Problemáticas de la enseñanza de la historia en Colombia: trabajar con la memoria en un país en guerra. Revista Reseñas, No 7. Argentina: Apehu.

Rodríguez, S. P. y Sánchez, O. (2009: b). Narrativa, memoria y enseñanza del conflicto armado colombiano: propuestas para superar las políticas del olvido y la impunidad. En: Adrián Serna (Compilador). El papel de la memoria en los laberintos de la justicia, la verdad y la reparación. Bogotá: Ipazud, U. Distrital.

Silva Briceño, O. (2010). Entre la memoria oficial y otras memorias: disputa de saber-poder en la enseñanza de las ciencias sociales. La primera violencia en Colombia. En: Emergencias de la memoria. Dos estudios sobre la infancia la escuela y la violencia. Bogotá: Ipazud, U. Distrital.

Sánchez Gómez, G. (2003). Guerras, memoria e historia. Bogotá: ICANH.

Todorov, T. (1995). Los abusos de la memoria. Paris: Paidós.

Vega, R. (1999). Historia: conocimiento y enseñanza. La cultura popular y la historia oral en el medio popular. Bogotá: Ediciones Antropos.

Vansina, J. (1982). La tradición oral y su metodología. En: Kiservo, J. Historia general de África, Volumen 1. España: Editoriales Tecnos-Unesco.

Vega, R. y Castaño, R. (1999). ¡Déjenos hablar! Profesores y estudiantes tejen historias orales en el espacio escolar. Bogotá D.C.: UPN-IDEP.

Zuluaga Garcés, O. L. y Marín Díaz, D. L. (2006). Memoria colectiva: memoria activa del saber pedagógico. En: Revista Educación y Ciudad. Bogotá, D.C.: IDEP. 